Pathophysiology of Haemostasis and Thrombosis

\title{
The Ecarin Clotting Time, a Universal Method to Quantify Direct Thrombin Inhibitors
}

\author{
Götz Nowak \\ Research Group ‘Pharmacological Haemostaseology', Medical Faculty, Friedrich Schiller University Jena, \\ Jena, Germany
}

\section{Key Words}

Ecarin clotting time - Meizothrombin generation test • Direct thrombin inhibitor $\cdot r$-Hirudin

\begin{abstract}
The ecarin clotting time (ECT) is a meizothrombin generation test that allows for precise quantification of direct thrombin inhibitors. The ECT has demonstrated its usefulness for more than 10 years in biochemical-pharmacological investigations as well as in clinical research and in the clinical routine. It has proved valuable especially as a drug-monitoring method in r-hirudin therapy. This test has been adjusted to clinical requirements by numerous modifications. Following the description of the biochemical background and the measuring principle of the ECT, this article gives a short survey of several modifications of the ECT for both preclinical and clinical use, e.g., for biochemical investigations, as a point-ofcare method and for cardiac surgery. Advantages and disadvantages of these methods are discussed.
\end{abstract}

Copyright $\odot 2004$ S. Karger AG, Basel

\section{This review is dedicated to the $\mathbf{8 0}^{\text {th }}$ birthday of Fritz Markwardt}

\section{Introduction}

In the late eighties of the past century, hirudin experienced a sudden turn towards a potential therapeutic use. Gene technology and the associated feasibility of producing and purifying large amounts of proteins outside the animal organism as well as preparing them for application in humans, have placed hirudin into the focus of interest in preclinical and phase I clinical investigations. A milestone has been set by the possibility of quantifying the effect of this direct serine proteinase inhibitor on thrombin by splitting chromogenic substrates [1]. Up to then, only global clotting methods like the activated partial thromboplastin time (aPTT) or the modified thrombin time (TT) had been used to analyse the efficacy of this substance class.

Using the knowledge that hirudin also interacts with meizothrombin, we succeeded in 1990/91 both in developing an antidote principle for thrombin inhibitors (TIs) [2] and in quantifying direct TIs, using the ecarin clotting time (ECT), with a precision never achieved until then. For the first time, an exact measuring method has become available that had been designed exclusively for this substance class and for a simple and fast quantitative measurement of direct TIs in blood or plasma without signifi-

Prof. Dr. Götz Nowak

Friedrich Schiller University Jena, Medical Faculty

Research Group 'Pharmacological Haemostaseology'

Drackendorfer Strasse 1, DE-07747 Jena (Germany)

Tel. +4936419325700, Fax +49364193257 02, E-Mail goetz.nowak@med.uni-jena.de
Fax +41613061234 E-Mail karger@karger.ch www. karger.com

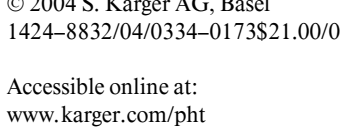


Fig. 1. Activation of prothrombin.

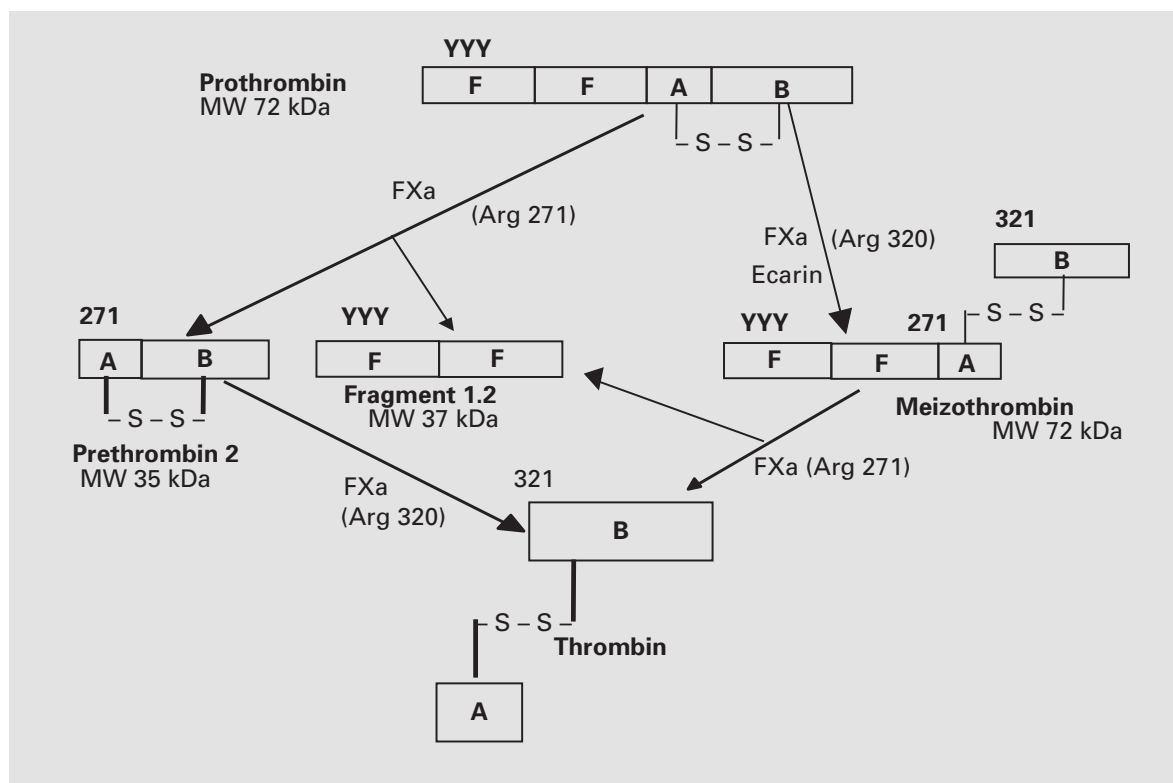

cant disturbing factors. In order to establish a broad application of the ECT, the first results of this method were published in 1993 [3]; a pilot paper followed in 1996 [4]. Thereafter, a multitude of application possibilities have been developed, demonstrating the uniqueness of this method both for fast bedside monitoring in clinical practice and for the quantification of antithrombin activity of direct synthetic TIs. Now, 10 years after the development of the ECT, a short review is given here. The increasing use of direct TIs in clinical practice requires a precise monitoring of patients. The ECT directly contributes to a safe application of this new drug class.

\section{Biochemical Background}

To start clotting in the ECT, a highly purified metalloprotease from the venom of the saw-scaled viper Echis carinatus is used that specifically activates prothrombin. In the final activation step of plasma coagulation, prothrombin is alternatingly activated to thrombin by prothrombinase at the Arg 271 and Arg 320 bonds in a twostep limited proteolysis. Prothrombinase is a membranebound complex of phospholipids, FXa, FVa and calcium ions. By cleaving prothrombin first at the Arg 271 bond, prethrombin 2 and fragment 1.2 of prothrombin arise, to which $\gamma$-carboxy groups are attached. Thus, the A-chain of prothrombin is significantly shortened. If the Arg 320 bond is cleaved first, an intermediate serine proteinase arises, meizothrombin, which has the same molecular weight as prothrombin and is already proteolytically active (fig. 1). This meizothrombin does not leave the prothrombinase complex and is activated to $\alpha$-thrombin in a second step while bound to the phospholipid surface, and will thereafter dissociate from the complex [5]. However, prethrombin 2 was shown to leave the activation complex and to be activated to $\alpha$-thrombin by other prothrombinase complexes. In the ECT, the direct prothrombin cleavage generates meizothrombin in soluble form, so that the ECT can also be called 'meizothrombin generation test'. The active centre of meizothrombin is difficult to reach for natural substrates. Therefore, the procoagulant activity of meizothrombin is relatively low, maximally $10 \%$ of the equimolar thrombin mass. Ecarin is able to cleave the Arg 320 bond of all prothrombin derivatives, which means ecarin can also activate prethrombin 1 to meizothrombin desF1, prethrombin 2 to thrombin, or PIVKA-prothrombin (A-carboxy-prothrombin circulating in plasma of patients treated with oral anticoagulants) to PIVKA-meizothrombin. This PIVKA-meizothrombin represents an optimal antidote substance for direct TI, especially hirudin [6]. Due to missing $\gamma$-carboxy groups in PIVKA-meizothrombin, its genuine clotting activity is further decreased. For the development of the ECT it was important that this specific, singular Arg 320 cleavage by ecarin was used for the reaction-rate-limiting generation of meizothrombin. Using a very small quantity of the enzyme, the ecarin-induced coagulation could be demon- 
strated to follow first-order reaction kinetics. Thus, a linear activity-time correlation is guaranteed when using this enzymatic reaction as a monitoring method. Ecarin is a very robust enzyme that maintains its full enzymatic activity for $24 \mathrm{~h}$ at room temperature. When stabilized with albumin, high-molecular-weight carbohydrate compounds or others, the enzyme can be stored refrigerated for several days. When frozen, the enzyme activity is maintained for at least 6 months.

\section{Measuring Principle}

The original ECT method was developed in 1991/92 and published for the first time in 1993 [3, 4]. It was demonstrated that the hirudin level can be determined by the ECT both in citrated and in heparinized blood. The ECT is completely insensitive to plasma heparin, as the heparin-AT complex cannot inhibit meizothrombin due to steric hindrance. It was also shown that AT alone does not inhibit meizothrombin for the same reason. Although ecarin does not require additional calcium ions for meizothrombin generation - and therefore the ECT can be performed in citrated or oxalated blood - calcium ions were added to the ecarin starting reagent in order to increase clotting strength. The mechanism underlying the monitoring method is very simple: due to the high ability of direct TIs, especially hirudin, but also synthetic lowmolecular-weight TIs, to inhibit meizothrombin, the permanently generated meizothrombin is neutralized as long as this TI is present in plasma. Only when all TIs are consumed in this reaction, can free meizothrombin be generated that will induce coagulation via fibrinogen cleavage. The permanent generation of meizothrombin depends on plasma prothrombin. For this reason, the quantity of ecarin has originally been chosen so as to achieve a reasonable clotting time $(\sim 50 \mathrm{~s})$ and, on the other hand, to ensure that sufficient prothrombin remains in the sample to intercept enough inhibitors when prolonging this clotting time. The calibration curve depicted in figure 2 shows that the clotting time in the therapeutic range of hirudin amounts to maximally $250 \mathrm{~s}$, representing a convenient and easily handled time interval in mechanical coagulometers as well as in automated analysers with a clotting channel. The sensitivity of the ECT for hirudin is $0.05 \mu \mathrm{g} / \mathrm{ml}$ in the lower detection limit. When scientific aspects require the measurement of lesser amounts of direct TI in plasma or body fluids, the lower detection limit can be decreased to $0.01 \mu \mathrm{g} / \mathrm{ml}$ by variation of the amount of added ecarin. However, in this case, the clot-

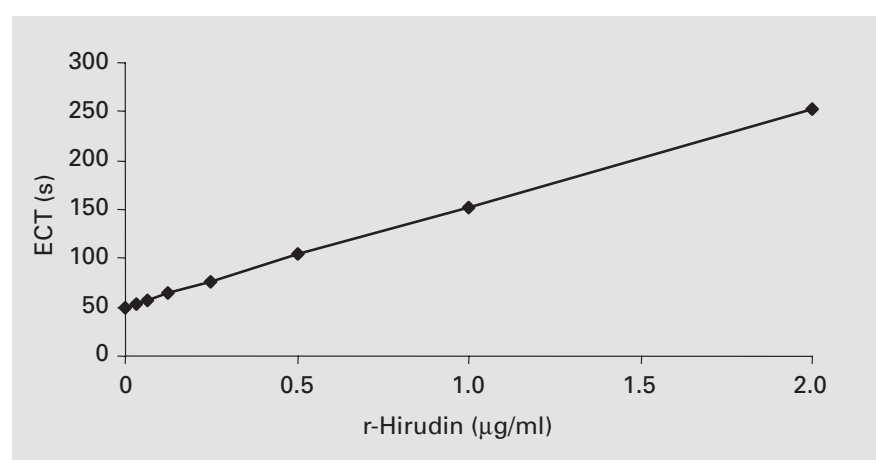

Fig. 2. r-Hirudin calibration curve in plasma (ECT, original method, mechanical coagulometer).

ting time in control plasma or blood amounts to 100 $120 \mathrm{~s}$. For use as a therapeutic drug monitoring method, these lower detection limits are of minor importance. In clinical routine, the measurement range between 0.1 and $2 \mu \mathrm{g} / \mathrm{ml}$ hirudin is relevant. Plasma levels of more than $2.5 \mu \mathrm{g} / \mathrm{ml}$ represent acutely toxic hirudin levels which seldom occur in clinical practice (with the exception of cardiac surgery) when using the ECT as the monitoring method for patient control and therapy management. Besides the advantages of the method mentioned so far, a further benefit is that the ECT is neither affected by oral anticoagulants nor by heparin. In clinical practice, this proved to be very favourable, especially when patients have to be shifted to an alternative anticoagulant. For instance, in thrombophilic states, the residual hirudin level must often be assayed in the case of concomitant oral anticoagulation. As ecarin also activates PIVKA-prothrombin to PIVKA-meizothrombin, measurement of hirudin in plasma is possible even with concomitant anticoagulant treatment.

\section{ECT Method}

\section{Original ECT Method}

To achieve the sample volume required by mechanical coagulometers, the same volume of TRIS buffer is added to a sample of citrated blood or citrated plasma. Measurement of the clotting time starts following the addition of an ecarin solution with a final concentration of 5 ecarin units $(\mathrm{EU}) / \mathrm{ml}$ in the coagulation assay. This ecarin solution is very stable and can be used for $24 \mathrm{~h}$ when stored at room temperature. Clotting time measurement in duplicate and calculation of average have been demonstrated to yield adequate accuracy. Variance of the ECT is less than 5\% (2.8-4.2\%). For therapeutic drug monitoring of hirudin and polyethylene glycol (PEG)-hirudin, calibration curves between 0 and $5 \mu \mathrm{g} / \mathrm{ml}$ hirudin/PEG-hirudin are established by addition of the highest inhibitor concentration to standard plasma (pooled plasma of at least 10 healthy volunteers) and 
Fig. 3. Concentration-clotting time curves for ECT, aPTT and TT.

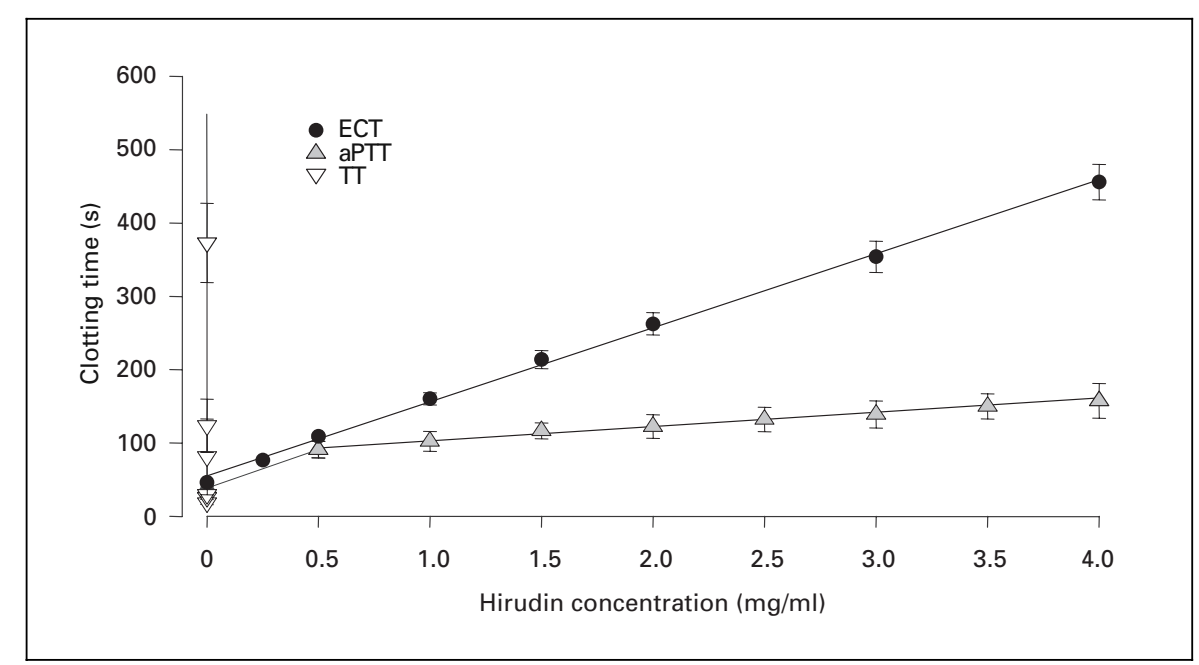

following successive 1:1 dilutions. Using such calibration plasma for comparative measurements of the ECT, aPTT and TT, it was demonstrated that in the whole calibration range of hirudin, the concentration-clotting time curve is linear in ECT (fig. 3). In contrast, the aPTT curve flattens with increasing hirudin levels; furthermore, it varies depending on the aPTT reagent used. The TT is not suited due to its very narrow measurement range. When using individual plasma of healthy volunteers or of patients without clotting disorders for calibration curves according to the same procedure as mentioned above, the baseline value of ECT in blood or plasma samples varies only slightly around a mean, and that individual concentrationclotting time curves show very similar slopes. Therefore, for therapeutic drug monitoring during Refludan ${ }^{\circledR}$ therapy or prophylaxis, a standard calibration curve can be used to achieve an accurate and reproducible measurement of hirudin levels. In further investigations, it was shown that the following parameters can affect ECT: temperature of the sample, change of buffer, $\mathrm{pH}$ of the sample [7]. For this ECT, which can also be performed as a bedside test, ball coagulometers of the Thrombotrack series (Behnk Elektronik, Norderstedt, Germany) or the KC series (Amelung) are used. Comparative measurements of the precision of the coagulometers have shown that coagulometers of the $\mathrm{KC}$ micro series provide the most exact results with the lowest variation. Another advantage of this KC micro series is the feasibility of a modified ECT (see 'Single-Step Hirudin Assay' below) where citrated whole blood can be poured directly into ECT reagent-coated cuvettes, making it an ultra-fast point-of-care testing (POCT) method for acute hirudin blood level determinations.

\section{Modified ECT Methods}

ECT Using Ecarin Reagent. For the measurement of plasma levels of direct TI, especially hirudin or PEG-hirudin in large numbers of samples, a so-called ecarin reagent is used in our laboratories, both in coagulation analysers and in mechanical coagulometers. This lyophilized reagent contains buffer, $\mathrm{CaCl}_{2}$ and $5 \mathrm{EU} / \mathrm{ml}$ ecarin like in the original ECT. As buffer, Hepes or TRIS is used. This reagent is to be dissolved prior to measurement, and then $100 \mu \mathrm{l}$ are added to 100 or $200 \mu \mathrm{l}$ of incubated plasma sample, depending on the coagulome- ter type. This ecarin reagent is especially suited for large numbers of samples, e.g., in clinical trials. The disadvantage when using this ecarin reagent in coagulation analysers with a clotting channel is that direct TI cannot be measured in blood, only in plasma. Clinically relevant blood levels can only be assayed using mechanical coagulometers. The ecarin reagent has the advantage of higher precision due to the optimal assay volume. Inter-individual and day-to-day variation have been improved. A typical calibration curve for the determination of the PEG-hirudin blood level is shown in figure 4.

Single-Step Hirudin Assay. For very fast and simple determination of hirudin with POCT quality, the single-step hirudin assay (SSHA) was developed as a modification of the original ECT. For the SSHA, coagulometer-specific cuvettes are coated (mechanical 1channel or 2-channel coagulometers are preferred). The amount of buffer-ecarin solution necessary for a single determination is lyophilized directly in the cuvettes that are thereafter tightly sealed with tinfoil. For determination of the hirudin level, the lyophilisate is dissolved in bidistilled water, and the clotting time measurement is started by the addition of $100 \mu \mathrm{l}$ citrated whole blood or citrated plasma. Due to its extreme simplicity, this bedside test can also be practiced by unskilled laboratory staff, e.g., in clinical emergency situations. For an ultra-fast blood level determination, especially coagulometers of the $\mathrm{KC}$ micro series are suited, as a dissolution of the lyophilized reagent is not necessary and the measurement can be done directly in citrated blood. In clinical routine, the coated cuvettes have demonstrated their advantages for the determination of ECT in mechanical coagulometers. The cuvettes have often been used, so that the experience gathered with many thousands of r-hirudin measurements is availble.

Thrombolytic Assessment System (Dry Card Method). For the measurement of direct TI using the ECT, the so-called dry card technology has proved to be suited as well. The provider of the appropriate equipment named 'thrombolytic assessment system' (Pharmanetics, Raleigh, N.C., USA) has also developed the dry card technology for the ECT measurement. In this device, ecarin and small iron chips are brought in contact with blood or plasma in a thin cavity of the test card. Beneath the test chamber, a magnet moves and induces the mixing of the sample and the iron chips. When clotting occurs, an 
inductive signal arises that is electronically registered as the clotting time. The amount of ecarin and buffer used in the test cards corresponds to the original ECT. The clotting time without antithrombin ranges between 48 and $55 \mathrm{~s}$. This dry card technology also possesses POCT quality and can be performed by unskilled laboratory personnel. However, due to the filling and measuring procedure in the test card, the precision is quite low. Calibration curves have only restricted practicability to achieve acceptable quantification. When using this technology in a clinical trial, satisfactory precision was only obtained after four-fold measurements and calculation of mean values. Most determinations of prophylactic blood levels of Refludan $(0.1-0.3 \mu \mathrm{g} / \mathrm{ml})$ did not provide reliable data; this method should thus be used only in emergency situations when no other method is available.

Modification of ECT for Very High Hirudin Blood Levels. In cardiosurgery, coronary bypass surgery or thoracic surgery, very high amounts of anticoagulants are necessary due to the low biocompatibility of the oxygenators and the large artificial extracorporeal surfaces. The resulting high blood levels $(2.5-3 \mu \mathrm{g} / \mathrm{ml}$ Refludan), at the limit of acute toxicity, cause very long clotting times (350-450 s) when using the original ECT. Due to the necessary close therapeutic drug monitoring, problems arise concerning a fast response to variations of blood level. Therefore, in the case of such high-dose hirudin administrations, the original ECT is modified and the withdrawn blood samples are immediately diluted 1:1 with human pooled plasma and are thereafter measured using one of the three methods mentioned above. With this procedure, on the one hand, effective measurement times are reached by halving the clotting times. On the other hand, by addition of standard plasma, the possible problem of prothrombin deficiency is avoided (see below). Using this modified ECT procedure recommended for cardiac surgery, toxic blood levels can be precisely measured and controlled as well.

Another Modification of ECT for Cardiosurgical Indications. For hirudin monitoring in cardiosurgery, both a whole blood method and a method using plasma have been modified $[8,9]$. In this case, significantly higher amounts of ecarin have been used. According to the device used (mechanical coagulometer or coagulation analyser with clotting channel), the baseline value of the ECT ranges between 18 and $38 \mathrm{~s}$. The amount of ecarin in the coagulation assay varies between 6.25 and $18 \mathrm{U} / \mathrm{ml}$. Methods using very high amounts of ecarin have been designed for the measurement of high hirudin blood levels. Calibration curves are linear up to high hirudin concentrations $(10 \mu \mathrm{g} / \mathrm{ml})$. A clear disadvantage of these methods is their significantly increased sensitivity for decreased prothrombin levels. When using automated coagulation analysers, the ECT can only be measured in plasma; thus, it cannot be performed as a POCT procedure. Pre-analytical centrifugation in order to obtain plasma makes this method useless for cardiac surgery, because the ECT results are not available quickly enough after blood withdrawal. The advantage of a relatively short measurement time in this modified ECT, most of all in the case of high hirudin blood levels, is abolished by the necessary centrifugation.

ECT for the Measurement of Direct TI in Urine. For pharmacological investigations in preclinical trials, but also for clinical use, a determination of direct TI or their metabolites in urine is possible using a modification of the ECT. For that purpose, $20 \mu \mathrm{l}$ urine are mixed with $60 \mu \mathrm{l}$ buffer (TRIS or barbiturate buffer) and are added to $100 \mu \mathrm{l}$ human pooled plasma. As in the original method, mechanical coagulometers are used. After $2 \mathrm{~min}$ of incubation at $37^{\circ} \mathrm{C}$, the ECT measurement is started by adding $20 \mu \mathrm{l}$ of ecarin reagent $(5 \mathrm{EU} / \mathrm{ml})$.

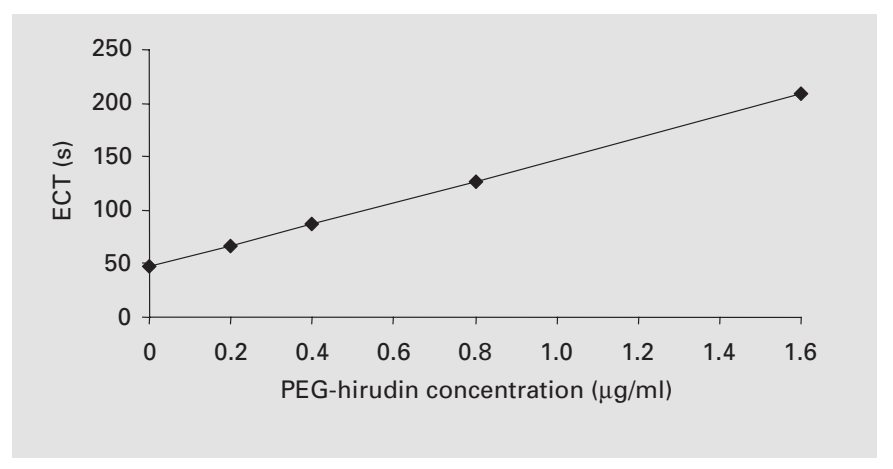

Fig. 4. PEG-hirudin calibration curve in blood (ECT using reagent, mechanical coagulometer).

By generating a calibration curve using blank urine spiked with 0.02 and $1 \mu \mathrm{g} / \mathrm{ml}$ urine of the direct TI, precise detection of direct TI as anti-meizothrombin activity becomes possible. Other body fluids (saliva, filtered gastro-intestinal contents, peritoneal fluid, cell lysates or homogenates of organs and others) can be investigated as well, following the same procedure. It has proven favourable to limit the amount of these fluids to $10 \mu \mathrm{l}$ in order to get exact results. In biochemical investigations, the amount of active direct TI or their metabolites present in fractions of separation or purification procedures can be determined in the same way.

\section{Results}

\section{ECT in Preclinical/Animal-Experimental Studies}

Calibration curves obtained from rat, rabbit and dog blood were very similar to those made using human blood or plasma. This enabled us to use the standard ECT for experiments with animals in pharmacokinetic and doseresponse investigations. The ECT gained particular importance in pharmacokinetic evaluation of new direct TI. Not only hirudin, but also PEG-hirudin, NAPAP, PPACK, PEGylated synthetic TI and other natural TI have been biochemically and pharmacologically characterized using the ECT. As an example, pharmacokinetics of r-dipetalogastin II (rDipII) in rats are reported here.

The strongest direct TI of the tropical assassin bug Dipetalogaster maximus is DipII, a double-headed, bifunctional, slow tight-binding TI (MW: $79 \mathrm{kDa}$ ) that was recently recombinantly expressed [10]. The ECT has been used for the entire molecular biological and biochemical characterization. Due to its high sensitivity, the ECT could be used for detection throughout the biochemical purification process. rDipII has been pharmacokinetically analysed in rats. The blood level of rDipII following subcutaneous and intravenous administration is shown in 


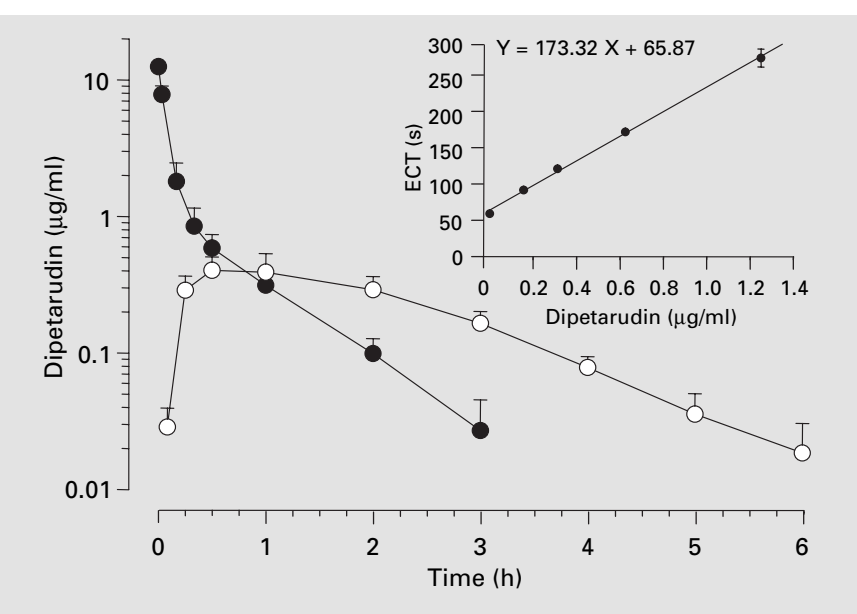

Fig. 5. Blood level of rDipII following subcutaneous ( $\square$ ) and intravenous $(-)$ administration of $1 \mathrm{mg} / \mathrm{kg}$ in rats. Results are mean of $\mathrm{n}=4$.

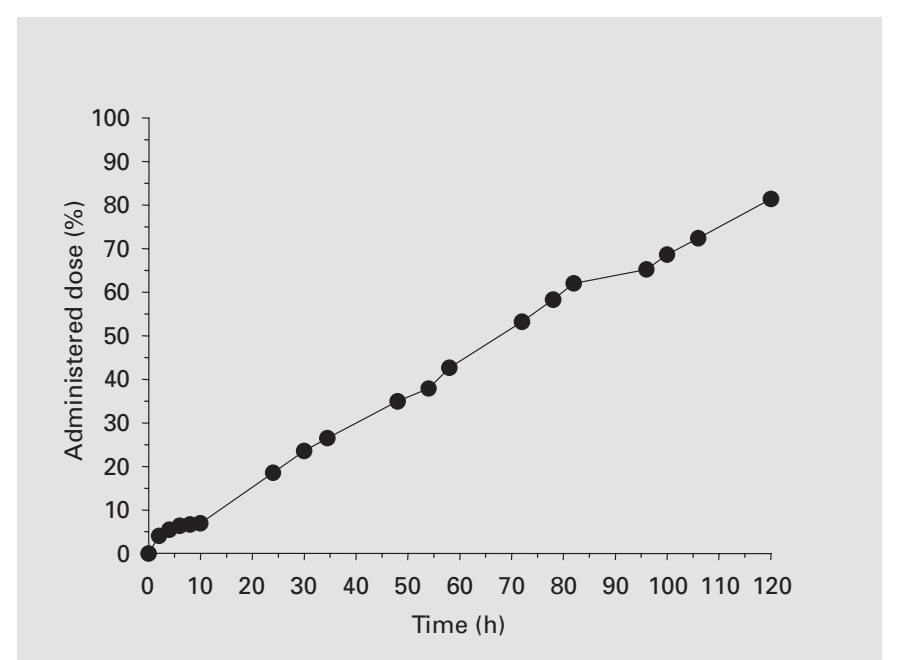

Fig. 6. Cumulative urinary excretion of rDipII expressed as percentage of administered dose, following intravenous injection of $1 \mathrm{mg} / \mathrm{kg}$ in rats (representative data of 1 out of 4 experiments).

figure 5. The calibration curve used for this determination is depicted in the insert. For an analysis of the renal elimination of this direct TI, the ECT has been slightly modified: to human standard plasma, both buffer and an equivalent amount of rat urine have been added. Using a calibration curve, the amount of rDipII in urine has been measured. The renal elimination of rDipII after intravenous bolus injection is shown for a period of 5 days in figure 6 .

\section{ECT as a Screening Method for Biochemical Characterization of Direct TI}

A multitude of synthetic direct TIs, but also new recombinant variants of natural TIs have been characterized in preclinical studies. ECT is suited for the biochemical classification of direct TI and differentiates between competitive inhibitors and tight-binding or irreversibly acting TI. In a special study [11], it has been shown that competitive synthetic TI, when added to human pooled plasma, have a non-linear course in the dose-clotting time curve. Only irreversible synthetic TI (e.g., PPACK) and slow tight-binding natural inhibitors (hirudin, rhodniin, dipetalogastin) show a strictly linear course of concentration-clotting time curve. Thus, interesting possibilities arise for a simple screening of substances. Standard ECT can also be used for biochemical characterization of the inhibitor type. Following molecular weight extension of synthetic TI using PEG coupling, not only an efficacy analysis of the substance in human plasma can be done, but also a specific synthesis work in order to influence reaction kinetics. As the ECT is a meizothrombin generation test, specificity investigations can generally also be done in order to differentiate between TI and meizothrombin inhibitors.

\section{ECT for Long-Term Drug Monitoring of Refludan in Haemodialysis}

The use of r-hirudin as an anticoagulant in haemodialysis (HD) is only possible when a simple and effective drug monitoring is available, as the r-hirudin blood level can be influenced by several variables. As some patients on chronic intermittent HD show considerable residual diuresis, these patients eliminate certain quantities of $r$ hirudin between the dialysis sessions. In contrast, anuric patients cannot eliminate r-hirudin. Only the amount of r-hirudin that is consumed by coagulation activation during extracorporeal therapy and due to prothrombotic disease must be compensated by the administration of a maintenance dose of r-hirudin prior to the dialysis session. The risk of Refludan cumulation and its side effects are very high in these cases. Therefore, these patients must be regularly monitored. As an example of an individual dose adjustment and long-term monitoring of a dialysis patient with Refludan, the long-term treatment of an HD patient is depicted in figure 7. Following an adjustment phase, the patient received a constant amount of Refludan leading to a nearly constant r-hirudin blood level. The largest variations of blood level occur between time points prior to HD and after HD. Therapeutic drug monitoring of patients suffering from chronic renal insuf- $\overline{178} \quad \overline{\text { Pathophysiol Haemost Thromb }}$ 2003/04;33:173-183
Nowak 
Fig. 7. Long-term monitoring (3 years) of r-hirudin (Refludan) in a patient (A.B., 31 years, female) on chronic renal replacement therapy.

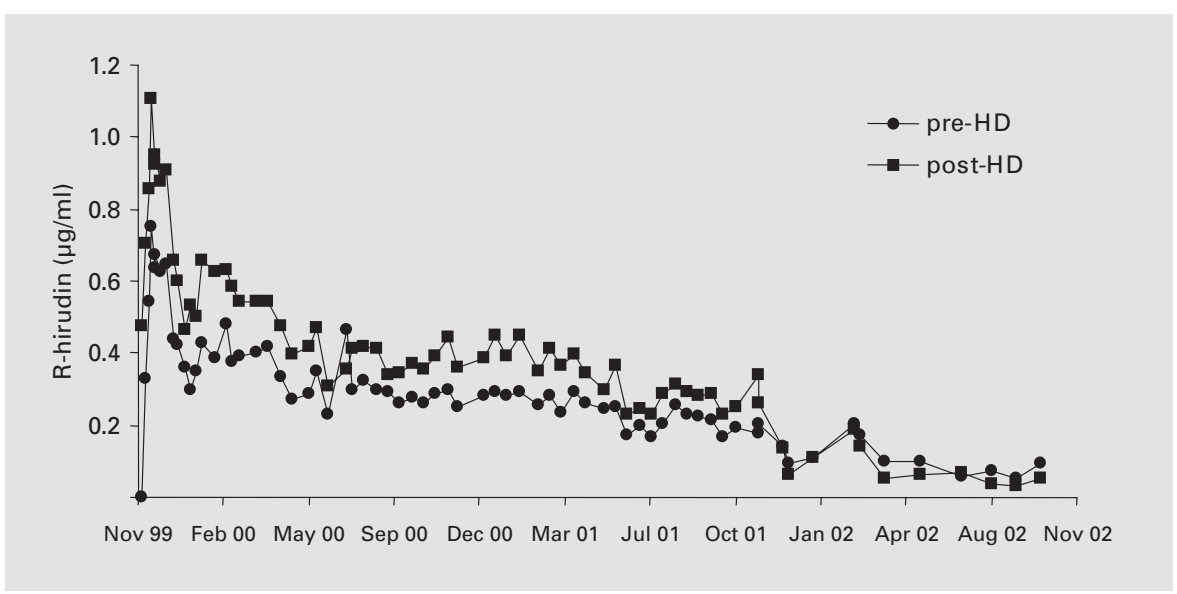

ficiency is extremely important. Patients with renal impairment who are treated with direct antithrombins must be monitored as well to avoid overdosing. A direct linear correlation between creatinine clearance and hirudin clearance in these patients is helpful. Using this correlation, a dose estimation or dose reduction can be done according to renal impairment. Nevertheless, individual drug monitoring for the prevention of an overdose or underdose is necessary, as individual factors can have an influence as well.

\section{ECT in Clinical Phase II Studies of Pharmacokinetics of PEG-Hirudin}

Prior to phase II/phase III studies of PEG-hirudin for several clinical indications, a safety study of PEG-hirudin was carried out in patients suffering from different degrees of renal impairment. The heavily delayed renal elimination of PEG-hirudin due to molecular weight enlargement of hirudin by PEG coupling requires detailed knowledge of the modified pharmacokinetics in renal impairment. In this study, several pharmacokinetic parameters were determined in 4 patient groups with different degrees of renal impairment (grouped according to decreased creatinine clearance). It has been clearly shown that in contrast to r-hirudin, PEG-hirudin does not undergo C-terminal degradation in proximal renal tubule cells [12]. Important data from this investigation on the pharmacokinetics and the course of the PEG-hirudin blood level are shown in figure 8. Surprisingly, the distribution of PEG-hirudin into the extravascular compartment was very slow, i.e. the pharmacokinetic mobility of hirudin is significantly decreased by PEGylation. A steady state between the extravascular and intravascular compart-

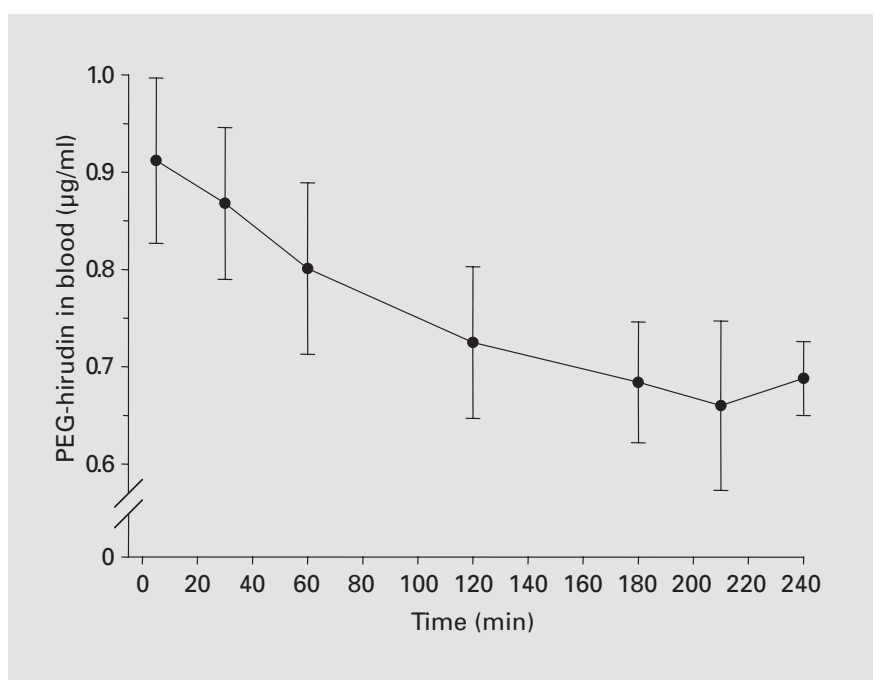

Fig. 8. Course of PEG-hirudin blood level during HD (mean \pm SD of $n=20$ patients) measured using ECT with ecarin reagent.

ments was reached only after $16-20 \mathrm{~h}$. Up to that time, more PEG-hirudin was found in the intravascular than in the extravascular space. Thus, very interesting aspects arise for the preferred usability of PEG-hirudin in chronic HD treatment. The maximum duration of an HD session of 5-6 h allows for a relatively high amount of PEG-hirudin to reach the intravascular space; thus, lower total amounts of PEG-hirudin are required for an effective HD compared with r-hirudin; PEG-hirudin can be dosed more precisely. 


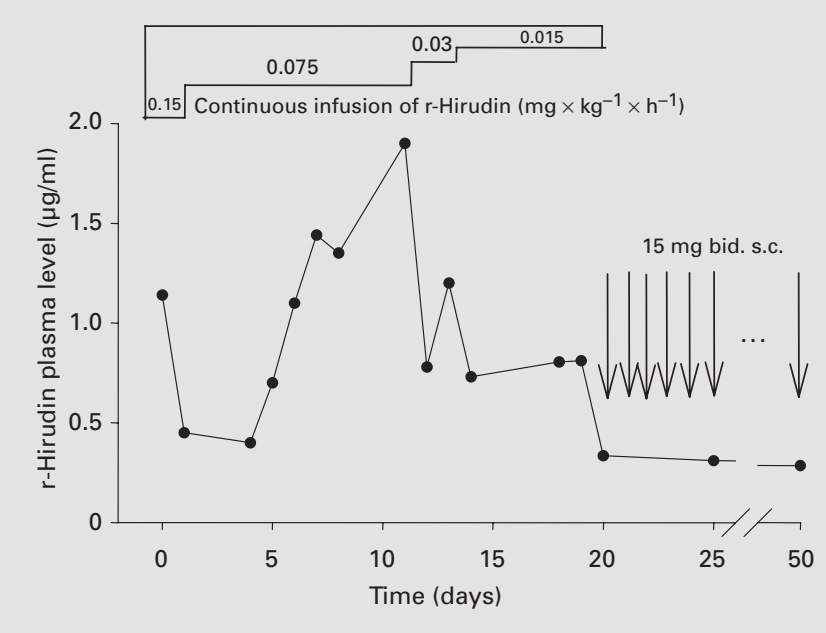

Fig. 9. Therapeutic drug monitoring using ECT. Long-term anticoagulation using r-hirudin (Refludan) in a stroke patient (P.K., 52 years, male) with manifest HIT-II after heparinization in knee replacement surgery. During the first days, relatively large amounts of r-hirudin were consumed. Three weeks later, the acquired coagulation disorder was controlled with subcutaneous hirudin application.

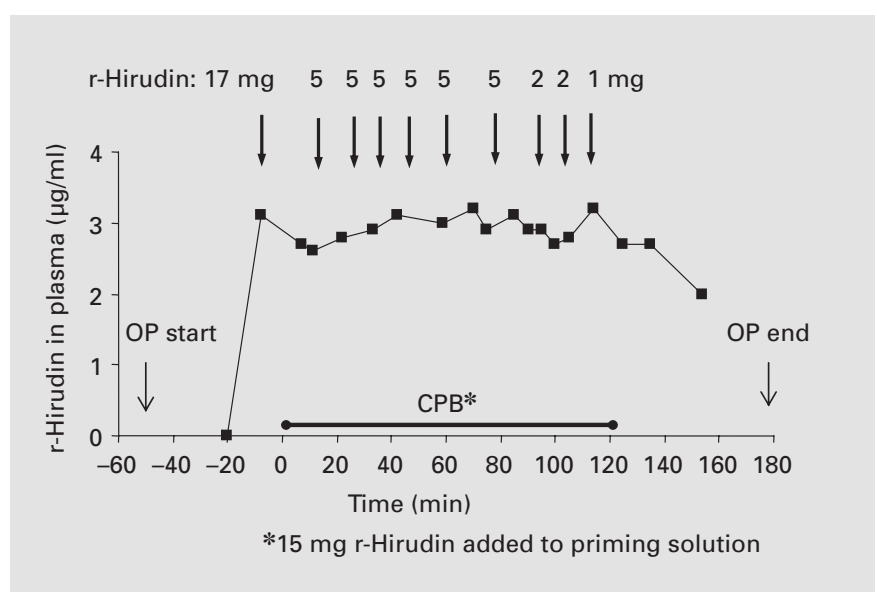

Fig. 10. Drug monitoring using ECT for r-hirudin anticoagulation during cardiopulmonary bypass surgery. By repeated r-hirudin bolus administration, a nearly constant r-hirudin plasma level of $2.5-3 \mu \mathrm{g} /$ $\mathrm{ml}$ was maintained. Overall, during the 3-hour-lasting surgery, the patient got $1 \mathrm{mg} / \mathrm{kg}$ r-hirudin [taken from ref. 18].

\section{ECT for Drug Monitoring of Refludan in Clinical Practice}

Both 'original' ECT and the modification using coated cuvettes (SSHA) have been used by many investigators in clinical practice. In particular, patients have been shifted to Refludan to prevent thromboembolism when heparininduced thrombocytopenia type II (HIT II) with clinical symptoms had been diagnosed. Figure 9 shows a longterm course of r-hirudin blood levels under Refludan anticoagulation in a patient with manifest HIT II. A co-medication with ASA must be avoided in order to prevent the known prolongation of bleeding times [13]. In a large number of orthopaedic patients with hip or knee replacement (indication for r-hirudin, Revasc), experience has shown that the r-hirudin blood levels should be determined in order to monitor these prophylactic r-hirudin levels both before and $2 \mathrm{~h}$ after subcutaneous r-hirudin administration. Two hours after $\mathrm{r}$-hirudin administration at the dose of $2 \times 15 \mathrm{mg}$ or $2 \times 20 \mathrm{mg}$, blood levels ranged between 0.2 and $0.3 \mu \mathrm{g} / \mathrm{ml}$ in patients with sound kidneys and decreased to values near the detection limit of the ECT until the next r-hirudin dose. Therapeutic drug monitoring in these orthopaedic patients is necessary as cumulating effects of r-hirudin can occur in patients with unrecognized renal dysfunction who receive long-term $\mathrm{r}$ hirudin prophylaxis of thromboembolism. A dose reduction of r-hirudin must be possible when the blood level is too high. In patients with renal impairment of more than $50 \%$ (according to creatinine clearance), the subcutaneous r-hirudin dose should be adapted to body weight and creatinine clearance in order to avoid an overdose.

\section{ECT for $r$-Hirudin Administration in Cardiosurgery}

Bedside monitoring during cardiosurgery of HIT II patients constitutes a special challenge to the ECT, especially as precision and simplicity are concerned. For this purpose, various modifications of the ECT have been published $[8,9]$. During cardiosurgery, compensated or decompensated consumptive reactions of the coagulation system can occur (most often a decrease of prothrombin or fibrinogen level). Therefore, the precision of the ECT in its original form is reduced. Furthermore, during cardiopulmonary bypass surgery, the r-hirudin level reaches $2.5-4 \mu \mathrm{g} / \mathrm{ml}$ in blood or plasma, so that long measurement times arise. The often used variation of ECT using increased ecarin amounts has caused not only a decreased sensitivity of the method, but also incorrect high hirudin levels due to reduced prothrombin levels or high prothrombin consumption during meizothrombin activation, respectively. However, the precision of the original method can be maintained when the withdrawn citrated whole blood $(100 \mu \mathrm{l})$ is directly added into cuvettes containing an aliquot amount of standard plasma $(100 \mu \mathrm{l})$. Due to this dilution, 1:1 of the withdrawn blood, r-hirudin levels are obtained that allow a fast and exact determina-
Nowak 
tion. Due to the normal amount of coagulation factors (most of all prothrombin) contained in standard plasma, the precision of ECT measurement is maintained. In figure 10, a typical course of such an r-hirudin blood level determination during cardiosurgery is shown.

\section{Discussion}

The ECT has proved to be a robust, simple and sufficiently sensitive method for measuring the concentration of direct TIs both in preclinical research and clinical routine. Its high specificity results from the fact that ecarin exclusively generates meizothrombin from animal and human prothrombin, and this meizothrombin having an already 'open' active centre interacts with direct, activesite-directed TI. The ECT cannot be used to detect states of disturbed coagulation, but has practical relevance only for therapeutic drug monitoring of antithrombin activity. By the increasing importance of meizothrombin activation for the prothrombin-thrombin pathway, the ECT can also be used for the screening of meizothrombin inhibitors, i.e. direct TI that preferentially inhibit meizothrombin. Specific meizothrombin inhibitors will have clinical importance because meizothrombin remains solid-phasebound in the prothrombinase complex during activation. Therefore, following in situ inhibition of meizothrombin, no liberation of $\alpha$-thrombin occurs, and no thrombin is generated during prothrombin activation. From recombinant variants of natural TI, different activities of meizothrombin and $\alpha$-thrombin inhibition have recently been identified [14]. Thus, the ECT, as a meizothrombin generation test, is also an original biochemical-pharmacological tool for the definition of a new specific inhibitor family, the 'meizothrombin inhibitors'. Meizothrombin also plays an important role in the activation of PARs, most of all in cancer cells [15]. However, further investigations must prove if this substance class has clinical relevance.

The limits of the ECT are reached when blood levels of prothrombin or fibrinogen are heavily decreased due to consumptive reactions or other severe states of disease. This strongly impairs the measuring principle and may generate wrong measurements. The dependence of quantitative measurability of direct TI on the current prothrombin level may lead to the conclusion that higher concentrations of direct TI, especially of hirudin, will generally cause wrong values. However, at a normal prothrombin level, the measurability of even higher hirudin concentrations is guaranteed. If the original or a modified ECT with higher ecarin amounts is used, an effective

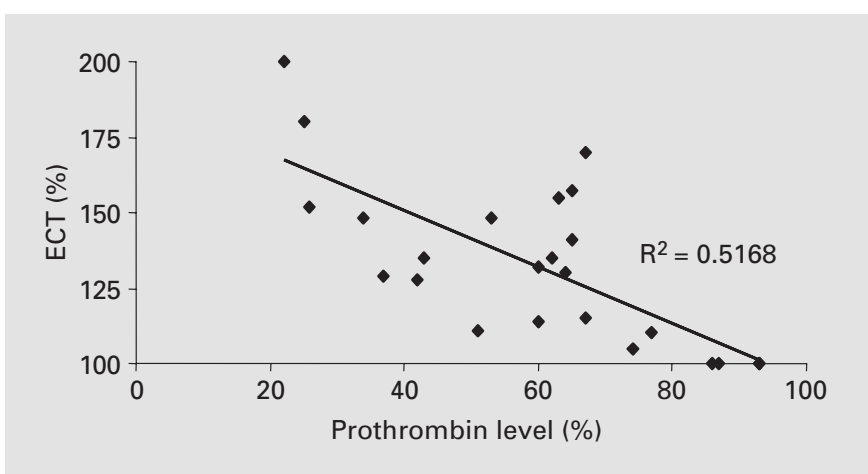

Fig. 11. Correlation between ECT prolongation and prothrombin level [data from ref. 16].

measuring principle during extreme measuring conditions in cardiosurgery with hirudin blood levels above $3.5 \mu \mathrm{g} / \mathrm{ml}$ is arguable.

Therefore, the modification for cardiosurgery (see 'Modification of ECT for Very High Hirudin Blood Levels' above) should be used in which, due to the dilution with pooled plasma, a sufficient amount of prothrombin is provided in the sample to compensate for consumptive reactions of fibrinogen. It cannot be excluded that the very high hirudin concentrations observed in cardiopulmonary bypass surgery are in fact too high. Experience with such operations using modified ECT methods have demonstrated that Refludan blood levels $>2.5 \mu \mathrm{g} / \mathrm{ml}$ are not necessary to keep the oxygenator circulation free from clots.

In an investigation using both a chromogenic substrate method and ECT in a large number of HIT II patients receiving Refludan, the variations in the results have been attributed to varying prothrombin levels. It has been concluded that ECT is inferior to the chromogenic thrombin substrate method [16]. Following critical reading of the paper, the incorrectly determined hirudin amounts that were supposed to result from decreased prothrombin levels have been closely examined, and a correlation of $\mathrm{R}^{2}=$ 0.52 between ECT prolongation and prothrombin level was found (fig. 11). This correlation shows that the differences between ECT and the chromogenic substrate method are not caused by variable amounts of prothrombin. Rather it must be assumed that the authors disregarded the fact that the chromogenic thrombin-derived substrate method is extremely influenced by the amount of antithrombin in the sample. As no quantitative determination of AT III levels was done in that study, an influence 
of AT III on the measured values must be assumed. Thrombin-derived chromogenic hirudin assays are also influenced by other anticoagulant substances that could be removed by special preliminary procedures (e.g., heparin-binding substances). Nevertheless, chromogenic assays represent an alternative method for the monitoring of blood or plasma levels of direct TIs. The lack of precision is arguably due to several disturbing factors, especially the AT III level.

A new method that uses the advantages of the ECT and compensates for its disadvantages has been established with the ecarin chromogenic assay (ECA) that has become available for the clinical routine [17]. In this assay, the measuring principle of ECT is used, i.e. ecarin is added to a plasma sample and meizothrombin generation is measured using a chromogenic substrate. Thus, the ECA represents a further development of the ECT that can be used both in automated analysers and in special low-cost manual analysers enabling a bedside measurement to be performed.

The original ECT has been designed so that blank values of clotting time in blood or plasma are below $1 \mathrm{~min}$, and the measurement of therapeutic blood levels does not take more than $3 \mathrm{~min}$. Duplicate measurements ensure a sufficient precision. The variation of calibration measurements of direct antithrombins does not exceed 5\%. However, as relatively high amounts of antithrombin increase the consumption of meizothrombin that must be generated from prothrombin contained in the sample, very high inhibitor levels can result in incorrect values. When the amount of ecarin in the sample is highly increased in order to decrease ecarin-induced clotting time as mentioned in the literature for some modifications [18], this measuring error becomes significantly higher due to the meizothrombin consumption. Practice has demonstrated that a possible fibrinogen deficiency does not cause measuring problems. The acute-phase protein fibrinogen is increased in many patients; an increase of up to $250 \%$ of the normal value has not impaired ECT determination. At a further increase of fibrinogen up to $300-400 \%$, measured hirudin levels have been reduced by about $10 \%$.

\section{Conclusion}

The ECT is a valuable monitoring method in the clinical routine. In the described specific methodical variants using $5 \mathrm{EU} / \mathrm{ml}$ ecarin (see 'Original ECT Method' and 'ECT Using Ecarin Reagent' above), no faulty ECT measurements of direct antithrombins have been observed.
This method has become established in preclinical research for many years and has been used for many in vitro and in vivo investigations of new potential antithrombotic substances. Excellent results of pharmacokinetic investigations of hirudin and hirudin derivatives have clearly demonstrated the superiority of the ECT over the aPTT, which is still recommended as a monitoring method by the manufacturers of r-hirudin variants. As the aPTT shows great variation (33-51 s) in the blank value in clinical practice, and furthermore, is affected by changes of coagulation variables in acute states of consumption, the precision of the aPTT is not comparable with that of the ECT. The ECT is exclusively a drug-monitoring method for direct antithrombins and will help to make them safer in their clinical use. By using ECT, a potential overdose can be detected as well. Special modifications of ECT have been developed for a safe monitoring of even very high TI concentrations as they are necessary in cardiosurgery, and thus provide good control of the important anticoagulation in this procedure. The continuous improvement of ECT has led to a clinically even better method, the ECA, with which the monitoring of direct TIs will become safer and easier as, in contrast to ECT, ECA can be used also in automated analysers. 


\section{References}

1 Griessbach U, Stürzebecher J: Assay of hirudin in plasma using chromogenic thrombin substrate. Thromb Res 1986;37:347-350.

$>2$ Nowak G, Bucha E: Ecarin-induced meizothrombin formation - an efficient antidote of hirudin. Thromb Res 1992;65:S157.

-3 Nowak G, Bucha E: A new method for the therapeutical monitoring of hirudin. Thromb Haemost 1993;69:1306.

$>4$ Nowak G, Bucha E: Quantitative determination of hirudin in blood and body fluids. Semin Thromb Hemost 1996;22:197-202.

$\checkmark 5$ Boskovic DS, Bajzar LS, Nesheim ME: Channeling during prothrombin activation. J Biol Chem 2001;276:28685-28693.

6 Nowak G, Bucha E: Prothrombin conversion intermediate effectively neutralizes toxic levels of hirudin. Thromb Res 1995;80:317-325.

7 Nowak G: Monitoring of the action of antithrombin agents by ecarin clotting time; in Pifarré R (ed): New Anticoagulants for the Cardiovascular Patient. Philadelphia, Hanley \& Belfus, 1997, pp 539-550.

$>8$ Pötzsch B, Hund S, Madlener K, Unkrig C, Müller-Berghaus G: Monitoring of recombinant hirudin - assessment of a plasma-based ecarin clotting time assay. Thromb Res 1997; 86:373-383.
9 Pötzsch B, Madlener K, Seelig C, Riess CF, Greinacher A, Müller-Berghaus G: Monitoring of r-hirudin anticoagulation during cardiopulmonary bypass - assessment of the whole blood ecarin clotting time. Thromb Haemost 1997; 77:920-925.

10 Mende K, Petoukhova O, Koulitchkova V, Schaub GA, Lange U, Kaufmann R, Nowak G: Dipetalogastin, a potent thrombin inhibitor from the bloodsucking insect Dipetalogaster maximus. CDNA cloning, expression and characterization. Eur J Biochem 1999;266: 583-590.

11 Novak G, Bucha E, Kossmehl A: Quantitative determination of synthetic thrombin inhibitors using Ecarin Clotting Time. Ann Hematol 1998;76(suppl 1):A51.

12 Esslinger HU, Bucha E, Pöschel K, Jansa U, Schindler S, Stein G, Nowak G: Pharmacokinetics of PEG-hirudin in subjects with various degrees of renal function. Ann Hematol 1998; 76(suppl 1):P278.

13 Breddin HK, Radziwon P, Esslinger HU: Thrombin inhibition enhances aspirin-induced bleeding time prolongation. Circulation 1996; 94:I-267.
14 Lopez ML, Nowak G: Special pharmacokinetics of dipetarudin suggests a potential antitumor activity of this thrombin inhibitor. Anticancer Drug 2004,15:145-149.

15 Kaufmann R, Zieger M, Tausch S, Henklein P, Nowak G: Meizothrombin, an intermediate of prothrombin activation, stimulates human glioblastoma cells by interaction with PAR-1type thrombin receptors. J Neurosci Res 2000; 59:643-648.

16 Lindhoff-Last E, Piechottka GP, Rabe F, Bauersachs R: Hirudin determination in plasma can be strongly influenced by the prothrombin level. Thromb Res 2000;100:55-60.

17 Lange U, Wiesenburg A, Olschewski A, Steinmetzer T, Nowak G, Bucha E: Ecarin chromogenic assay (ECA) - a new chromogenic assay useful for clinical monitoring of direct thrombin inhibitors like hirudin. Ann Hematol 2002; 81(suppl 1):A49.

18 Riess FC, Pötzsch B, Müller-Berghaus G: Recombinant hirudin as an anticoagulant during cardiac surgery; in Pifarré R (ed): New Anticoagulants for the Cardiovascular Patient. Philadelphia, Hanley \& Belfus, 1997, pp 197-221. 